Proyecciones Journal of Mathematics

Vol. 32, No 3, pp. 259-265, September 2013.

Universidad Católica del Norte

Antofagasta - Chile

\title{
Asymptotics for Klein-Gordon equation
}

\author{
Ana M. Marín \\ Universidad de Cartagena, Colombia \\ Rubén D. Ortiz \\ Universidad de Cartagena, Colombia \\ and \\ Joel A. Rodriguez Ceballos \\ Instituto Tecnológico de Morelia, Mexico \\ Received: September 2012. Accepted : May 2013
}

\begin{abstract}
We propose a simple method for constructing an asymptotic of an eigenvalue for the Klein-Gordon equation in the presence of a shallow potential well, reducing the initial problem to an integral equation and then by applying the method of Neumann series to solve it.
\end{abstract}

Keywords : Klein-Gordon equation, asymptotics, eigenfunctions.

Subjclass [2010] : 81Q15, 31A10. 


\section{Introduction}

In [5], we find the Klein-Gordon equation

$$
\Phi_{t t}-\Delta \Phi+m^{2} \Phi=0, \quad m>0,
$$

where $\Delta$ is the Laplacian in dimension $n$, perturbed by a potential $U=$ $U(x)$ to

$$
\Phi_{t t}-\Delta \Phi+m^{2} \Phi+U \Phi=0 .
$$

We look for the solution of the equation Phi in the form

$$
\Phi=\exp (i \omega t) \Psi(x)
$$

where $\omega$ is the frequency. If we replace PhiSi in Phi, then we obtain the equation

$$
\left(-\Delta+m^{2}+U\right) \Psi=E \Psi, \quad E=\omega^{2} .
$$

When $m=0$, we have the Schrödinger equation

$$
(-\Delta+U) \Psi=E \Psi
$$

that in the case when $U$ describes a shallow potential well (i.e., $U=$ $\left.\varepsilon V(x), V(x) \in C_{0}^{\infty}\left(R^{n}\right), \varepsilon \rightarrow 0\right)$, it has one eigenvalue $E_{0}=-\beta^{2}, \beta \in R$ below the essential spectrum $[0, \infty)$ with $\int_{R^{n}} V(x) d x \leq 0$ and the dimension $n$ of the configuration space is 1 or 2 . This was established for $n=1$ and in the radially symmetric case for $n=2$ in the famous book of Landau and Lifshitz [4] and it was demonstrated in the general case in dimension 2 by Simon [6]. Close results to the limit behavior of the resolvent can be found in [1], [3]. In [8], a different method was used for obtaining the asymptotics of the eigenfunctions.

It is based on a construction of eigenfunctions. It happens that this construction is elemental, when we pass to the momentum representation. Also, this method is efficient for the Schrödinger and Klein-Gordon equation.

The latter problems were studied by several authors (we mention, for example, $[2,3,4,5,6,7])$.

\section{Mathematical formulation}

The mathematical formulation of the problem under consideration is as follows. We look for non trivial solutions $\Phi \in L^{2}(R)$, of the problem

$$
-\Phi_{x x}(x)+m^{2} \Phi(x)+\varepsilon V(x) \Phi(x)=E \Phi(x)
$$


where $\varepsilon \rightarrow 0$ and $V$ is such that $\int_{-\infty}^{\infty} V(x) d x \leq 0$ and $V$ has compact support, then $V(x)=0$ for $|x|>R$ with $R$ sufficiently big. Given that the operator of multiplication by a function of compact support is compact in $L^{2}$, the continuous spectrum of (2.1) coincides with the continuous spectrum of the non perturbed equation $(\varepsilon=0)$ and the last is the interval $\left[m^{2}, \infty\right)$. We prove the following theorem.

Theorem 2.1. If $\int_{-\infty}^{\infty} V(x) d x<0$. Then the problem (2.1) has an eigenvalue

$$
E=-\beta^{2}+m^{2}+O\left(\varepsilon^{3}\right),
$$

where

$$
\beta=-\frac{\varepsilon}{2} \int_{-\infty}^{\infty} V(x) d x+\frac{\varepsilon^{2}}{32 \pi} \int_{\Gamma} \int_{-\infty}^{\infty} \int_{-\infty}^{\infty} V(s) V(t) e^{i(s-t) \zeta} d t d s \frac{d \zeta}{\zeta}+O\left(\varepsilon^{3}\right)
$$

is the solution of the secular equation for $\beta$ (5.8). The contour $\Gamma$ is defined by the equation (5.2).

\section{Heuristic considerations}

Denoting the Fourier transform by

$$
\tilde{V}(p)=(2 \pi)^{-1 / 2} \int_{R} e^{-i p x} V(x) d x
$$

As in [8], the formulas that appear in the Theorem 2.1 are based on the following heuristic reasoning: For $E=-\beta^{2}+m^{2}$, the solution of (2.1) for $|x|>R$ is given by $\Phi(x) \sim e^{-\beta|x|}$. We obtain a function that is "almost constant", when $\beta \rightarrow 0$. Since being "almost constant", its Fourier transform is a sequence of delta type when $\beta \rightarrow 0$.

Hence, $\tilde{\Phi}(p) \sim \tilde{V}(p) /\left(p^{2}+\beta^{2}\right)$. Therefore the Fourier transform of $\Phi$ is approximately equal to

$$
\tilde{\Phi}(p)=\frac{A(p)}{p^{2}+\beta^{2}}
$$




\section{Reduction to an integral equation}

Taking the Fourier transform in the equation L1, we obtain $\tilde{\Phi}(p)\left(p^{2}+\beta^{2}\right)=-\frac{\varepsilon}{\sqrt{2 \pi}} \int_{-\infty}^{\infty} W\left(p, p^{\prime}\right) \tilde{\Phi}\left(p^{\prime}\right) d p^{\prime}$ at $p \in(-\infty, \infty)$. Here $W\left(p, p^{\prime}\right)$ is given by $\mathrm{W}\left(\mathrm{p}, \mathrm{p}^{\prime}\right)=\widetilde{V}\left(p-p^{\prime}\right)$, where the tilde denotes the Fourier transform.

\section{Demonstration of the Theorem 2.1}

Proof. Taking $E=-\beta^{2}+m^{2}, \beta \rightarrow 0+$, we look for a solution of the equation (4) in the form

$$
\tilde{\Phi}(p)=\frac{A(p)}{p^{2}+\beta^{2}} .
$$

Substituting (5.1) in (4), we obtain $\mathrm{A}(\mathrm{p})=-\frac{\varepsilon}{\sqrt{2 \pi} \int_{-\infty}^{\infty} W\left(p, p^{\prime}\right) \frac{A\left(p^{\prime}\right)}{\left(p^{\prime 2}+\beta^{2}\right)} d p^{\prime}}$.

Denoting $\Omega$ the space of analytic functions on $B_{1}$ and continuous on $\overline{B_{1}}$ with the standard norm of the supreme, $\|\varphi\|=\sup _{z \in B_{1}} \varphi(z)$ for all $\varphi \in \Omega$. Here $B_{1} \equiv\{z \in C,|\Im z|<1\}$.

The zeros of the expression ${p^{\prime}}^{2}+\beta^{2}$ are $\pm z_{\beta}$, where $\mathrm{z}_{\beta}=i \beta$.

We change the contour of integration in the complex plane such that the zero $z=z_{\beta}$ is bounded away from it. Suppose that $A(z)$ belongs to $\Omega$ (then we prove that this is in fact the case) and introduce the contour

$$
\Gamma:=(-\infty,-1] \cup\left\{p+i q: p^{2}+q^{2}=1, q>0\right\} \cup[1, \infty) .
$$

If $\beta<1 / 2$, then $z_{\beta}$ is located below $\Gamma$. By the Cauchy residue theorem, equation (5) takes the form

$$
\beta\left(-A(p) \sqrt{2 \pi}-\varepsilon \int_{\Gamma} \frac{W(p, \zeta) A(\zeta) d \zeta}{\zeta^{2}+\beta^{2}}\right)=\pi \varepsilon W\left(p, z_{\beta}\right) A\left(z_{\beta}\right)
$$

Define the operator $T_{\beta}: \Omega \rightarrow \Omega$ by the formula

$$
\left[T_{\beta} \varphi(\zeta)\right](z)=\frac{1}{\sqrt{2 \pi}} \int_{\Gamma} \frac{W(z, \zeta) \varphi(\zeta) d \zeta}{\zeta^{2}+\beta^{2}}, z \in \Omega
$$

$\left[T_{\beta} \varphi(\zeta)\right](z) \in \Omega$ (the integrand is analytic) and $T_{\beta}$ is well-defined. $\left[T_{\beta} \varphi(\zeta)\right](z)$ is analytic in $\beta:|\beta / \zeta|<1$ for $z \in \Gamma$ and

$$
\frac{1}{\zeta^{2}+\beta^{2}}=\frac{1}{\zeta^{2}} \sum_{m=0}^{\infty} \frac{(-1)^{m}}{\zeta^{2 m}} \beta^{2 m} \text {. }
$$


Furthermore, $T_{\beta}$ is bounded. In fact, $\left\|T_{\beta} \varphi\right\|=\sup _{z \in B_{1}}\left|\frac{1}{\sqrt{2 \pi}} \int_{\Gamma} \frac{W(z, \zeta) \varphi(\zeta) d \zeta}{\zeta^{2}+\beta^{2}}\right|$ $\leq \frac{1}{\sqrt{2 \pi}} \sup _{z \in B_{1}} \int_{\Gamma} \frac{|W(z, \zeta) \varphi(\zeta) d \zeta|}{\left|\zeta^{2}+\beta^{2}\right|} \leq \frac{C\|\varphi\|}{\sqrt{2 \pi}} \int_{\Gamma} \frac{|d \zeta|}{\left|\zeta^{2}+\beta^{2}\right|}$ for some constant $C$. Hence, $\varepsilon\left\|T_{\beta}\right\|<1$ for $\varepsilon$ sufficiently small. Now from equation (5.3) we have

$$
-\sqrt{2 \pi} \beta\left[\left(1+\varepsilon T_{\beta}\right) A(\zeta)\right](z)=\pi \varepsilon W\left(z, z_{\beta}\right) A\left(z_{\beta}\right) .
$$

Given that $\varepsilon T_{\beta}$ is a contraction operator, we can invert the operator $\left(1+\varepsilon T_{\beta, \zeta \rightarrow z}\right)$

$$
A(z)=-\sqrt{\frac{\pi}{2}} \frac{\varepsilon}{\beta}\left[\left(1+\varepsilon T_{\beta, \zeta \rightarrow z}\right)^{-1} W\left(\zeta, z_{\beta}\right)\right](z) .
$$

We have a uniformly convergent series of analytic functions in $z$ on $B_{1}$. Hence $A(z)$ is analytic in $z \in B_{1}$. Suppose $A\left(z_{\beta}\right)=1$, as we can prove it later. Evaluating at $z=z_{\beta}$, from equation (5.7) we obtain the secular equation for $\beta$ :

$$
\beta=-\sqrt{\frac{\pi}{2}} \varepsilon\left[\left(1+\varepsilon T_{\beta, \zeta \rightarrow z}\right)^{-1} W\left(\zeta, z_{\beta}\right)\right]\left(z_{\beta}\right) .
$$

Consider the function

$$
F(\beta, \varepsilon)=\beta+\sqrt{\frac{\pi}{2}} \varepsilon\left[\left(1+\varepsilon T_{\beta, \zeta \rightarrow z}\right)^{-1} W\left(\zeta, z_{\beta}\right)\right]\left(z_{\beta}\right) .
$$

Substituting the Neumann series instead of $\left(1+\varepsilon T_{\beta}\right)^{-1}$ in equation (5.9), we obtain

$$
F(\beta, \varepsilon)=\beta+\sqrt{\frac{\pi}{2}} \varepsilon \sum_{l=0}^{\infty}(-1)^{l} \varepsilon^{l}\left[T_{\beta, \zeta \rightarrow z}^{l} W\left(\zeta, z_{\beta}\right)\right]\left(z_{\beta}\right) .
$$

$\left[T_{\beta, \zeta \rightarrow z}^{l} W\left(\zeta, z_{\beta}\right)\right]\left(z_{\beta}\right)$ is analytic in $\beta$. Then the function $F(\beta, \varepsilon)$ is analytic in each argument, and by Hartogs' theorem, it is analytic in $C^{2}$. Also, $F(0,0)=0,\left[\partial_{\beta} F\right](0,0)=1$. By the implicit function theorem, the solution $\beta(\varepsilon)$ for $\beta$ of the secular equation (5.8) there exists and is unique. We have $\left[\partial_{\varepsilon} F\right](0,0)=(\pi / 2)^{1 / 2} W(0,0),\left[\partial_{\beta, \varepsilon}^{2} F\right](0,0)=0,\left[\partial_{\beta}^{2} F\right](0,0)=0$,

$$
\left[\partial_{\varepsilon}^{2} F\right](0,0)=-\frac{1}{2} \int_{\Gamma} \frac{W(0, \zeta) W(\zeta, 0) d \zeta}{2 \zeta} .
$$

So we have up to the second order terms the expansion

$$
F(\beta, \varepsilon)=\beta+\varepsilon \sqrt{\frac{\pi}{2}} W(0,0)-\frac{\varepsilon^{2}}{4} \int_{\Gamma} \frac{W(0, \zeta) W(\zeta, 0) d \zeta}{2 \zeta}+\cdots
$$


The secular equation (5.8) is equivalent to $F(\beta, \varepsilon)=0$, i.e., we obtain bet. Also, from equation $\mathrm{Ab}$ and bet we have $A\left(z_{\beta}\right)=1$. Theorem 2.1 is proved.

\section{Acknowledgements}

The authors express their gratitude to CONACYT-México, Programa de Mejoramiento del Profesorado (PROMEP)-México and Universidad de Cartagena for financial support.

\section{References}

[1] Albeverio, S., Gesztesy, F., Høegh-Krohn, R., and Holden, H. : Solvable Models in Quantum Mechanics, Springer-Verlag, New York, (1988).

[2] Chadam, J. M. : The asymptotic behavior of the Klein-Gordon equation with external potential, J. Math. Anal. Appl., 31, pp. 334-348, (1970).

[3] Gadyl'shin, R. : Local perturbations of the Schrödinger operator on the axis, Theo. Math. Phys., 132, pp. 976-982, (2002).

underwater ridge.

[4] Landau, L. D., and Lifshitz, E. M. : Quantum mechanics, Pergamon, London, (1958).

[5] Schonbek, T. P. : On Inverse Scattering for the Klein-Gordon Equation, Transactions of the American Mathematical Society, 166, pp. 101-123, (1972).

[6] Simon, B. : The bound state of weakly coupled Schrödinger operator in one and two dimensions, Ann. Phys., 97, pp. 279-288, (1976).

[7] Synge, J. L. : A Klein-Gordon Model Particle, Proceedings of the Royal Society of London. Series A, Mathematical and Physical Sciences, Vol. 283, No. 1392, pp. 14-17, (1965).

[8] Zhevandrov, P., and Merzon, A. : Asymptotics of eigenfunctions in shallow potential wells and related problems, Amer. Math. Soc. Transl, Ser. 2. 208 (53), pp. 235-284, (2003). 
Ana M. Marín,

Universidad de Cartagena,

Facultad de Ciencias Exactas y Naturales,

Sede Piedra de Bolivar, Avenida del Consulado,

Cartagena de Indias,

Bolivar,

Colombia

e-mail : amarinr@unicartagena.edu.co

\section{Rubén D. Ortíz,}

Universidad de Cartagena,

Facultad de Ciencias Exactas y Naturales,

Sede Piedra de Bolivar,

Avenida del Consulado,

Cartagena de Indias,

Bolivar,

Colombia

e-mail : rortizo@unicartagena.edu.co

and

Joel A. Rodríguez-Ceballos

Instituto Tecnologico de Morelia,

Michoacán,

Mexico

e-mail : joel@ifm.umich.mx 